\title{
Association between Serum Ferritin and Circulating Oxidized Low-density Lipoprotein Levels in Patients with Type 2 Diabetes
}

\author{
YUKIO IKEDA, TADASHI SUEHIRO, SHIGEO YAMANAKA*, YOSHITAKA KUMON*, HIROSHI TAKATA, \\ SHOJIRO INADA, NAOKO OGAMI, FUMIAKI OSAKI, MARI INOUE, KAORU ARII AND KOZO HASHIMOTO \\ Department of Endocrinology, Metabolism and Nephrology, Kochi Medical School, Kochi University, Kohasu, Oko-cho, Nankoku, \\ Kochi 783-8505, Japan \\ *Laboratory Medicine, Kochi Medical School, Kochi University, Kohasu, Oko-cho, Nankoku, Kochi 783-8505, Japan
}

\begin{abstract}
The oxidative modification of low-density lipoproteins (LDL) plays a central role in the initiation and acceleration of atherosclerosis. Iron plays a part in the formation of highly toxic free radicals such as hydroxide and superoxide anions, which can induce lipid peroxidation. We investigated whether serum iron status was associated with circulating oxidized LDL (oxLDL) levels in type 2 diabetic patients, in whom oxidative stress and susceptibility to lipid oxidation were supposedly increased. Serum ferritin levels were significantly correlated with plasma oxLDL concentrations in both male and female patients ( $p<0.02$ and $p<0.05$, respectively). No correlation was detected between ferritin and LDL-cholesterol (LDL-C) concentrations despite the close correlation between LDL-C and oxLDL concentrations $(p<0.0001)$. Stepwise regression analysis showed that ferritin concentration was an independent positive determinant of oxLDL level, in addition to triglyceride concentration, body mass index and sex. This is the first report to show that serum ferritin is associated with circulating oxLDL levels in patients with type 2 diabetes. Further work is required to establish a causative link between iron excess and the development of diabetic vascular complications.
\end{abstract}

Key words: Oxidized LDL, Iron, Ferritin, Type 2 diabetes

(Endocrine Journal 53: 665-670, 2006)

THE oxidative modification of low-density lipoproteins (LDL) plays a central role in the initiation and acceleration of atherosclerosis $[1,2]$. Oxidized LDL (oxLDL), which is generally considered to be generated in vessel walls, exerts several proatherogenic effects, including increased synthesis and secretion of adhesion molecules, monocyte chemotaxis and adhesion, cytotoxicity to endothelial cells, enhanced foam cell formation, and increased smooth muscle cell proliferation $[3,4]$. oxLDL has also been identified in the circulation and has been shown to be associated with coronary artery disease $[5,6]$, although it remains unclear whether the plasma oxLDL originates in the arte-

Received: January 18, 2006

Accepted: June 21, 2006

Correspondence to: Yukio IKEDA, M.D., Ph.D., Department of Endocrinology, Metabolism and Nephrology, Kochi Medical School, Kochi University, Kohasu, Oko-cho, Nankoku, Kochi 783-8505, Japan rial wall or is generated in the circulation.

It is well known that iron is closely associated with oxidative stress. Iron takes part in the formation of highly toxic free radicals such as hydroxide and superoxide anions, which can induce lipid peroxidation [7]. Materials from atherosclerotic plaques have been shown to both contain iron and stimulate hydroxylradical generation and lipid peroxidation $[8,9]$. In accordance with this, it has been reported that iron chelation blocks oxidation of LDL [10]. Unused iron is stored as ferritin molecules to ensure neutralization, and iron must be in its free form to act as a prooxidant agent. Iron can be released from ferritin by the action of a reducing agent that converts $\mathrm{Fe}^{3+}$ into $\mathrm{Fe}^{2+}$, and its release is accelerated when antioxidant concentrations are decreased [7]. Thus, ferritin can act both as a molecule that protects against iron toxicity and as a source of iron that induces oxidative stress [11].

Considerable evidence suggests that hyperglycemia is associated with excess oxidation $[12,13]$. It has been 
shown that iron-induced LDL oxidation is enhanced by glucose in a time- and concentration-dependent manner in vitro [14]. Hyperglycemia is also associated with mild but chronic inflammation, which can accelerate the oxidation of lipoproteins [15]. Further, nonenzymatic glycation of LDL makes it more susceptible to oxidation [16]. Despite the accumulated data on the relation between iron, hyperglycemia and lipoprotein oxidation in vitro, there is no data showing an association between iron status and oxLDL in the diabetic state. We therefore studied the relationship between circulating oxLDL levels and ferritin concentrations in patients with type 2 diabetes.

\section{Materials and Methods}

We recruited 144 patients with type 2 diabetes based on the 1999 World Health Organization criteria (74 males and 70 females; mean age \pm SD: $64 \pm 12$ years). These patients had no ketoacidosis, renal failure (serum creatinine $\geq 2.0 \mathrm{mg} / \mathrm{dL}$ ), liver disorders, or recent history of cardiovascular disease. Subjects being treated with iron or drugs known to affect lipoprotein oxidation (e.g. vitamin $\mathrm{E}$, vitamin $\mathrm{C}$, or probucol) were excluded from the study. Subjects who had any history of blood transfusion were also excluded. None of the subjects had excessive alcohol intake. All subjects were Japanese and resided in the same area (Kochi Prefecture, Japan), and gave written informed consent to participate prior to the study.

Patients were considered to have diabetic nephropathy if they had a urine protein concentration of $>0.30$ $\mathrm{g} / \mathrm{L}$ at two or more consecutive measurements separated by an interval of 4 weeks or more. Patients were considered to have coronary artery disease if they had any one of the following: 1) acute myocardial infarction or confirmed non-acute myocardial infarction based on serial readings of baseline and annual electrocardiograms; 2) coronary artery disease requiring bypass surgery or angioplasty; or 3) angina confirmed by angiography or by ischemic changes found during non-invasive testing.

Fasting blood samples were collected from the subjects and transferred to tubes with or without EDTA$2 \mathrm{Na}$ for plasma and sera, respectively. Plasma and sera were immediately isolated by centrifugation and stored frozen at $-80^{\circ} \mathrm{C}$ until use. Plasma oxLDL concentration was measured by a sandwich enzyme-linked immunosorbent assay using monoclonal anti-oxidized LDL antibody (mAb-4E6) [5] and anti-apolipoprotein B antibody (Oxidized LDL ELISA kit, Mercodia AB, Uppsala, Sweden). Serum ferritin concentration was determined using commercially available kits (ECLusys Ferritin, Roche Diagnostics, Basel, Switzerland). High sensitive C-reactive protein (hsCRP) concentration was measured using latex-enhanced immunonephelometry (N High Sensitivity CRP, Dade Behring, Deerfield, IL, USA).

All data are presented as the mean $\pm \mathrm{SD}$. Comparisons of variables between two groups were performed using the Student's unpaired $t$-test. Correlations between different parameters were determined by Pearson product-moment correlation coefficients. Because serum ferritin concentrations showed skewed distributions in both sexes they were log-transformed (logtransformed ferritin). Multiple regression analysis was conducted using a stepwise method and a software program on a personal computer (StatView, version 4.5; Abacus Concepts, Berkeley, CA, USA). Sex (male = 1 , female $=2$ ), age, body mass index (BMI), duration of diabetes, $\mathrm{HbA}_{1 \mathrm{C}}$, log-transformed ferritin, hemoglobin, triglycerides, high density lipoprotein (HDL)cholesterol, uric acid, hsCRP, hypertension (absent $=$ 1 , existing $=2$ ), current smoking status (no $=1$, yes $=$ 2 ), and statin use (no $=1$, yes $=2$ ) were incorporated as independent variables for analysis of plasma oxLDL concentration. Variables were considered independent determinants if the $F$ value was confirmed to be more than 4.0. Statistical significance was defined as $p<0.05$.

\section{Results}

The mean plasma oxLDL concentration in the study population was $51.5 \pm 15.3 \mathrm{U} / \mathrm{L}$ (range 19.6-95.0 U/L), and it was significantly higher in female patients than male patients (Table 1). Serum ferritin concentration was significantly higher in male patients than female patients $(225.4 \pm 163.3$ vs $95.6 \pm 97.3 \mathrm{ng} / \mathrm{mL}, p<0.0001$, respectively) (Table 1). Log-transformed ferritin concentration was significantly correlated with plasma oxLDL concentration in both male and female patients (Fig. 1-a). No correlation was detected between logtransformed ferritin concentration and LDL-cholesterol (LDL-C) concentration (Fig. 1-b), although the LDL-C concentration was closely correlated with oxLDL concentration in both male $(\mathrm{r}=0.664, p<0.0001)$ and fe- 
Table 1. Clinical characteristics of the study subjects

\begin{tabular}{lccl}
\hline \multicolumn{1}{c}{ Parameter } & Male & Female & \multicolumn{1}{c}{$p$} \\
\hline$n$ & 74 & 70 & \\
Age (years) & $63.6 \pm 12.5$ & $64.7 \pm 12.2$ & N.S. $^{\mathrm{a}}$ \\
BMI $\left(\mathrm{kg} / \mathrm{m}^{2}\right)$ & $24.3 \pm 5.4$ & $25.1 \pm 5.3$ & N.S. $^{\mathrm{a}}$ \\
Diabetes duration (years) & $14.8 \pm 9.1$ & $13.8 \pm 8.4$ & N.S. $^{\mathrm{a}}$ \\
HbA $_{\mathrm{CC}}(\%)$ & $7.0 \pm 1.1$ & $7.5 \pm 1.4$ & $0.0173^{\mathrm{a}}$ \\
Total cholesterol (mg/dL) & $174 \pm 40$ & $198 \pm 35$ & $0.0002^{\mathrm{a}}$ \\
Triglycerides (mg/dL) & $129 \pm 77$ & $130 \pm 72$ & N.S. $^{\mathrm{a}}$ \\
HDL-cholesterol (mg/dL) & $47 \pm 13$ & $54 \pm 17$ & $0.0099^{\mathrm{a}}$ \\
LDL-cholesterol (mg/dL) & $101 \pm 36$ & $119 \pm 30$ & $0.0015^{\mathrm{a}}$ \\
Hemoglobin (g/dL) & $13.3 \pm 2.0$ & $12.4 \pm 1.7$ & $0.0035^{\mathrm{a}}$ \\
Creatinine (mg/dL) & $0.91 \pm 0.53$ & $0.79 \pm 0.52$ & N.S. $^{\mathrm{a}}$ \\
Uric acid (mg/dL) & $5.5 \pm 1.4$ & $4.7 \pm 1.4$ & $0.0007^{\mathrm{a}}$ \\
High sensitive CRP (mg/dL) & $0.30 \pm 0.71$ & $0.22 \pm 0.34$ & N.S. $^{\mathrm{a}}$ \\
Therapy (diet/OHA/insulin) & $14 / 38 / 22$ & $7 / 26 / 37$ & $0.0158^{\mathrm{b}}$ \\
Statin use & $12(16.2 \%)$ & $30(42.9 \%)$ & $0.0004^{\mathrm{b}}$ \\
Hypertension & $43(58.1 \%)$ & $44(62.9 \%)$ & N.S. $^{\mathrm{b}}$ \\
Current smoking & $47(63.5 \%)$ & $14(20.0 \%)$ & $<0.0001^{\mathrm{b}}$ \\
Persistent proteinuria & $17(23.0 \%)$ & $12(17.1 \%)$ & N.S. $^{\mathrm{b}}$ \\
Coronary artery disease & $14(18.9 \%)$ & $15(21.4 \%)$ & N.S. $^{\mathrm{b}}$ \\
Oxidized LDL (U/L) & $48.3 \pm 14.3$ & $54.8 \pm 15.7$ & $0.0101^{\mathrm{a}}$ \\
Ferritin (ng/mL) & $225.4 \pm 163.3$ & $95.6 \pm 97.3$ & $<0.0001^{\mathrm{a}}$ \\
\hline Data are & & &
\end{tabular}

Data are presented as number (percentage) or mean \pm SD. Statistical differences between males and females were tested using an unpaired Student's $t$ test $^{\mathrm{a}}$ or a chi-square test ${ }^{\mathrm{b}}$. N.S.: not significant. Hypertension: diastolic blood pressure $\geq 90 \mathrm{mmHg}$, systolic blood pressure $\geq 140 \mathrm{mmHg}$, and/or use of antihypertensive medication. OHA: oral hypoglycemic agent.

male patients $(\mathrm{r}=0.525, p<0.0001)$. Log-transformed ferritin concentration also showed a significantly positive correlation to oxLDL levels adjusted for serum LDL-C concentration (oxLDL/LDL-C ratio) in male $(\mathrm{r}=0.258, p<0.05)$ but not in female patients $(\mathrm{r}=$ 0.081 , not significant). Stepwise regression analysis of the independent determinants for oxLDL concentration showed that log-transformed ferritin concentration was a positive determinant of oxLDL concentration in addition to triglycerides, sex and BMI (Table 2-a). If we incorporated LDL-C concentration as an independent variable into this model, only LDL-C $(\beta=0.606, F=$ 99.083) and triglyceride concentrations $(\beta=0.364, F=$ 35.658) remained significant for oxLDL concentration (total $R^{2}=0.511, p<0.0001$ for the model) because of the close relationship between oxLDL and LDL-C concentrations. Another stepwise regression analysis in which the oxLDL/LDL-C ratio was used as a dependent variable instead of oxLDL also showed logtransformed ferritin to be an independent contributor, as were triglycerides and hemoglobin concentration (Table 2-b).
Table 2. Stepwise multiple regression analysis for plasma oxLDL concentration (a) or oxLDL/LDL-C ratio (b) in diabetic patients

\begin{tabular}{lrrr}
\hline \multicolumn{1}{c}{ Variable } & $\beta$ & $F$ & $p$ \\
\hline (a) oxLDL concentration & & & \\
Triglycerides & 0.299 & 15.434 & 0.0001 \\
Sex & 0.317 & 13.836 & 0.0003 \\
BMI & 0.247 & 10.642 & 0.0014 \\
Log ferritin & 0.233 & 6.605 & 0.0113 \\
(b) oxLDL/LDL-C ratio & & & \\
Triglycerides & 0.381 & 23.808 & $<0.0001$ \\
Hemoglobin & -0.232 & 8.705 & 0.0034 \\
Log ferritin & 0.180 & 5.063 & 0.0261 \\
Hypertension & 0.159 & 3.376 & 0.0684 \\
High sensitive CRP & 0.154 & 3.173 & 0.0772 \\
\hline
\end{tabular}

Stepwise multivariate regression analysis was performed. Parameters that were incorporated as independent variables were as follows: $\operatorname{sex}($ male $=1$, female $=2)$, age, BMI, duration of diabetes, $\mathrm{HbA}_{1 \mathrm{C}}$, log-transformed ferritin, hemoglobin, triglycerides, HDLcholesterol, uric acid, high sensitive CRP, hypertension (absent = 1 , existing =2), smoking (no=1, yes $=2$ ), statin use (no=1, yes $=2$ ).

The $F$ value for the inclusion and exclusion of variables was set at 4.0 at each step. $\beta$ : partial regression coefficient.

(a) Total $R^{2}=0.300, p<0.0001$ for the model. (b) Total $R^{2}=$ $0.228, p<0.0001$ for the model.

Female patients with persistent proteinuria had significantly higher ferritin levels compared to those without $(146.4 \pm 143.9$ vs $85.1 \pm 82.5 \mathrm{ng} / \mathrm{mL}$, respectively, $p<0.05)$ although the difference did not reach significance in male patients $(258.5 \pm 194.9$ vs $215.5 \pm 153.3$ $\mathrm{ng} / \mathrm{mL}$, respectively). There was no difference in ferritin levels between patients with and without coronary artery disease in both sexes (data not shown).

\section{Discussion}

Tissue iron excess has been shown to contribute to the production of reactive oxygen species and oxidative tissue damage. Body iron stores, expressed as serum ferritin levels, have been suggested to be associated with atherosclerosis $[17,18]$, although the epidemiological data are still conflicting [19]. The most widely accepted hypothesis explaining these associations is that a high ferritin level is associated with higher oxidative stress, which can accelerate the oxidation of lipoproteins. In the present study, we found significant positive correlations between serum ferritin concentrations and circulating oxLDL levels in both male 
Male

(a)

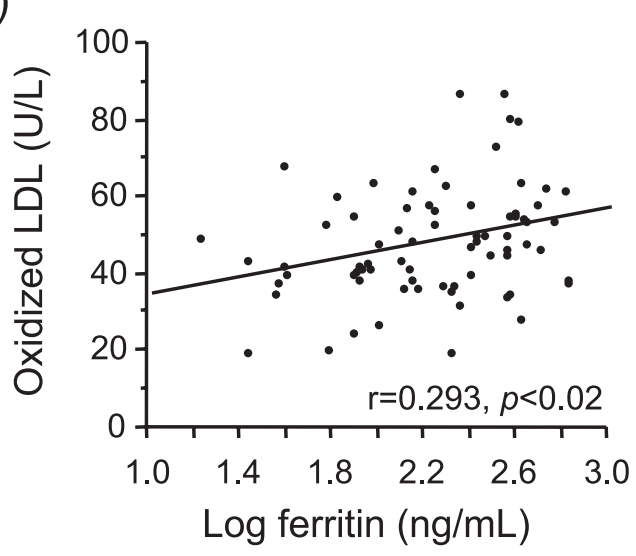

(b)

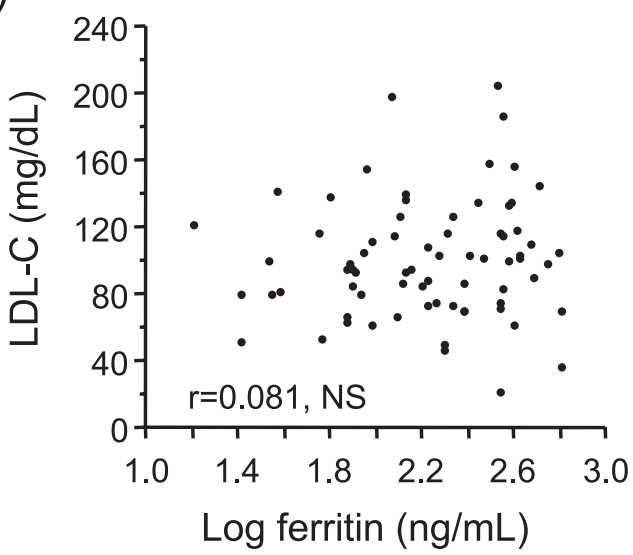

Female
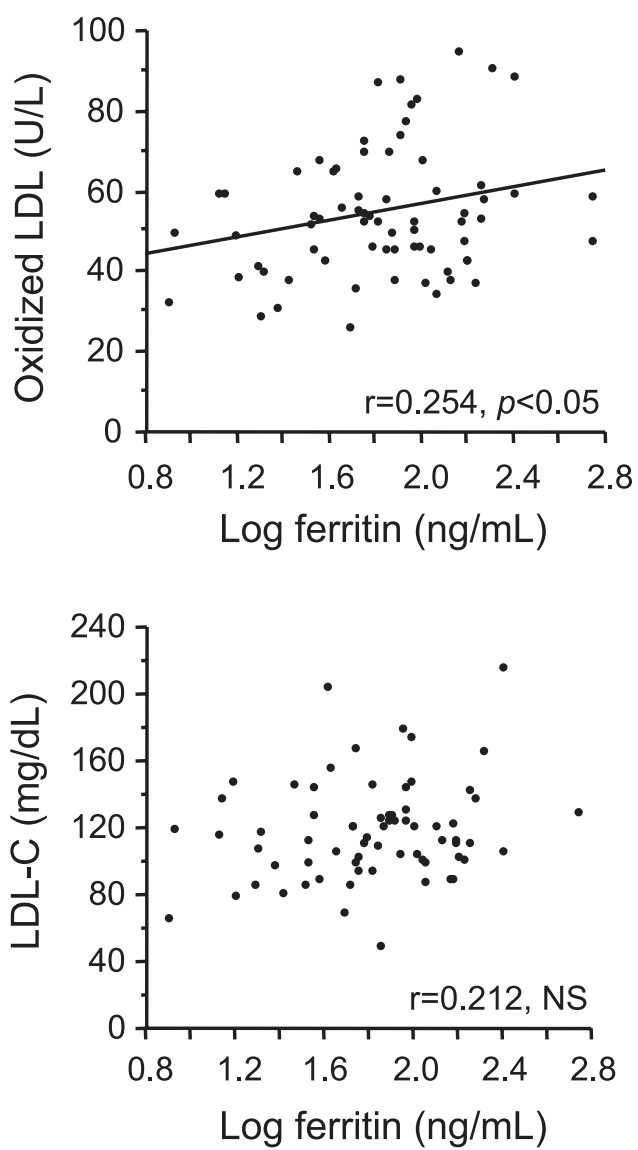

Fig. 1. Correlations between log-transformed ferritin and plasma oxidized LDL (a) and LDL-cholesterol (b) concentrations in type 2 diabetic patients.

Single linear univariate correlations were evaluated by Pearson's correlation coefficient.

and female patients with type 2 diabetes. The oxLDL level was closely correlated with LDL-C concentration; however, no significant correlation was observed between ferritin and LDL-C levels. Further, multiple regression analysis for oxLDL confirmed that ferritin concentration was a significant independent contributor, as were sex, BMI, and triglycerides, which are well-known risk factors for atherosclerosis. This is the first report showing a positive association between serum ferritin and circulating oxLDL levels in diabetic patients, in whom oxidative stress and susceptibility to lipid oxidation are supposedly increased.

There are a few clinical reports in which associations between ferritin levels and LDL oxidation parameters have been examined [20,21]. For example, Iribarren et al. [20] reported that ferritin concentrations were not associated with susceptibility to LDL oxidation (dura- tion of the lag phase after inducing oxidation with hemin) or autoantibodies against malondialdehyde (MDA)-modified LDL in a general population including $8-10 \%$ diabetic patients. On the other hand, Brouwers et al. [21] found that serum ferritin concentration was independently associated with plasma MDA concentration and the oxLDL/LDL-C ratio, which may reflect the proportion of oxidatively modified LDL to total LDL, in healthy male but not female Caucasian subjects. This result seems to be consistent with our present result, although Brouwers et al. did not demonstrate a direct association between serum ferritin concentration and circulating oxLDL level. Also, among our male patients log-transformed ferritin concentration was positively correlated with oxLDL/LDL-C ratio. In addition, multiple regression analysis confirmed that log-transformed ferritin was an independent con- 
tributor to the oxLDL/LDL-C ratio as well as oxLDL concentration. It remains unclear why both the present findings and those of Brouwers et al. did not show a significant association between oxLDL/LDL-C ratio and serum ferritin levels in female subjects. Among our patients, triglycerides and HDL-cholesterol concentrations, both of which are major components of the metabolic syndrome, were correlated with the oxLDL/LDL-C ratio more closely in females $(\mathrm{r}=0.528$, $p<0.0001$ and $\mathrm{r}=-0.460, p<0.0001$, respectively) than males $(\mathrm{r}=0.289, p<0.05$ and $\mathrm{r}=-0.104$, not significant, respectively). Such differences in the impacts of other contributing factors on the oxLDL/LDL-C ratio could be part of the reason for the different associations between serum ferritin levels and the oxLDL/LDL-C ratio between sexes.

There are some reports suggesting a significant influence of tissue iron excess on the development of diabetic nephropathy and vascular dysfunction [22, 23]. In the present study, serum ferritin levels in patients with nephropathy were higher than in patients without. Although ferritin is a source of iron that induces oxidative stress, it can also act as a molecule that protects against iron toxicity by neutralizing unused free iron [11]. Because iron can be released from ferritin by the action of a reducing agent that converts $\mathrm{Fe}^{3+}$ into $\mathrm{Fe}^{2+}$, its release is accelerated when antioxidant concentrations are decreased [7]. In diabetic patients who are thought to have increased oxidative stress and decreased antioxidant capacity, ferritin may act as an undesirable molecule because iron release from ferritin is promoted under these conditions.

It should be noted that this was a cross-sectional study and that the number of subjects participating was too small to arrive at definitive conclusions. Therefore, a large-scale prospective study is required to establish a link between serum ferritin concentration and the development of vascular complications in diabetic patients.

\section{References}

1. Navab M, Berliner JA, Watson AD, Hama SY, Territo MC, Lusis AJ, Shih DM, Van Lenten BJ, Frank JS, Demer LL, Edwards PA, Fogelman AM (1996) The Yin and Yang of oxidation in the development of the fatty streak. A review based on the 1994 George Lyman Duff Memorial Lecture. Arterioscler Thromb Vasc Biol 16: 831-842.

2. Witztum JL (1994) The oxidation hypothesis of atherosclerosis. Lancet 344: 793-795.

3. Berliner JA, Heinecke JW (1996) The role of oxidized lipoproteins in atherogenesis. Free Radic Biol Med 20: 707-727.

4. Steinberg D (1997) Low density lipoprotein oxidation and its pathobiological significance. J Biol Chem 272: 20963-20966.

5. Holvoet P, Vanhaecke J, Janssens S, Van de Werf F, Collen D (1998) Oxidized LDL and malondialdehydemodified LDL in patients with acute coronary syndromes and stable coronary artery disease. Circulation 98: 1487-1494.

6. Holvoet P, Mertens A, Verhamme P, Bogaerts K, Beyens G, Verhaeghe R, Collen D, Muls E, Van de Werf F (2001) Circulating oxidized LDL is a useful marker for identifying patients with coronary artery disease. Arterioscler Thromb Vasc Biol 21: 844-848.

7. Halliwell B (1993) The role of oxygen radicals in human disease, with particular reference to the vascular system. Haemostasis 23 (suppl 1): 118-126.
8. Smith C, Mitchinson MJ, Aruoma OI, Halliwell B (1992) Stimulation of lipid peroxidation and hydroxylradical generation by the contents of human atherosclerotic lesions. Biochem J 286: 901-905.

9. Thong PS, Selley M, Watt F (1996) Elemental changes in atherosclerotic lesions using nuclear microscopy. Cell Mol Biol 42: 103-110.

10. Abdalla DS, Campa A, Monteiro HP (1992) Low density lipoprotein oxidation by stimulated neutrophils and ferritin. Atherosclerosis 97: 149-159.

11. Juckett MB, Balla J, Balla G, Jessurun J, Jacob HS, Vercellotti GM (1995) Ferritin protects endothelial cells from oxidized low density lipoprotein in vitro. $\mathrm{Am}$ J Pathol 147: 782-789.

12. Wolff SP, Jiang ZY, Hunt JV (1991) Protein glycation and oxidative stress in diabetes mellitus and ageing. Free Radic Biol Med 10: 339-352.

13. Nourooz-Zadeh J, Tajaddini-Sarmadi J, McCarthy S, Betteridge DJ, Wolff SP (1995) Elevated levels of authentic plasma hydroperoxides in NIDDM. Diabetes 44: 1054-1058.

14. Mowri HO, Frei B, Keaney JF Jr (2000) Glucose enhancement of LDL oxidation is strictly metal ion dependent. Free Radic Biol Med 29: 814-824.

15. Berliner JA, Navab M, Fogelman AM, Frank JS, Demer LL, Edwards PA, Watson AD, Lusis AJ (1995) Atherosclerosis: basic mechanisms. Oxidation, inflammation, and genetics. Circulation 91: 2488-2496. 
16. Bowie A, Owens D, Collins P, Johnson A, Tomkin GH (1993) Glycosylated low density lipoprotein is more sensitive to oxidation: implications for the diabetic patient? Atherosclerosis 102: 63-67.

17. Salonen JT, Nyyssonen K, Korpela H, Tuomilehto J, Seppanen R, Salonen R (1992) High stored iron levels are associated with excess risk of myocardial infarction in eastern Finnish men. Circulation 86: 803-811.

18. Kiechl S, Willeit J, Egger G, Poewe W, Oberhollenzer F (1997) Body iron stores and the risk of carotid atherosclerosis: prospective results from the Bruneck study. Circulation 96: 3300-3307.

19. Danesh J, Appleby P (1999) Coronary heart disease and iron status: meta-analyses of prospective studies. Circulation 99: 852-854.

20. Iribarren C, Sempos CT, Eckfeldt JH, Folsom AR
(1998) Lack of association between ferritin level and measures of LDL oxidation: the ARIC study. Atherosclerosis Risk in Communities. Atherosclerosis 139: 189-195.

21. Brouwers A, Langlois M, Delanghe J, Billiet J, De Buyzere M, Vercaemst R, Rietzschel E, Bernard D, Blaton V (2004) Oxidized low-density lipoprotein, iron stores, and haptoglobin polymorphism. Atherosclerosis 176: 189-195.

22. Fernandez-Real JM, Lopez-Bermejo A, Ricart W (2002) Cross-talk between iron metabolism and diabetes. Diabetes 51: 2348-2354.

23. Wilson JG, Lindquist JH, Grambow SC, Crook ED, Maher JF (2003) Potential role of increased iron stores in diabetes. Am J Med Sci 325: 332-339. 